\title{
Relevancia de los intangibles para la valoración de las acciones de las empresas en el mercado: evidencias desde el contexto argentino
}

\author{
The relevance of intangibles for the valuation of companies \\ shares in the market: Evidence from the Argentine context \\ Cecilia Rita Ficco*1, Eliana Werbin², Margarita Díaz², \\ María Begoña Prieto Moreno ${ }^{3}$ \\ ${ }^{1}$ Universidad Nacional de Río Cuarto, Argentina \\ ${ }^{2}$ Universidad Nacional de Córdoba, Argentina \\ ${ }^{3}$ Universidad de Burgos, España
}

Recibido el 9 de mayo de 2019; aceptado el 17 de agosto de 2020

Disponible en Internet el: 7 de septiembre de 2020

\section{Resumen}

El objetivo de este trabajo es analizar la influencia de la información sobre activos intangibles y capital intelectual en la valoración de las acciones de las empresas que cotizan en el MCA, donde no se han constatado trabajos publicados de esta naturaleza. El análisis se realiza aplicando un modelo de construcción propia, basado en Ohlson (1995). Los datos corresponden al período 2009-2018 y han sido recolectados directamente de fuentes originales. Se obtuvieron de los estados financieros anuales consolidados de las empresas y de los registros de negociación de sus acciones en el Mercado de Valores de Buenos Aires. Los resultados revelan que los activos intangibles identificables, el capital humano y el estructural poseen relevancia valorativa, lo que contribuye a acrecentar la evidencia existente en torno al papel de los intangibles en la valoración externa de las empresas, la cual es particularmente limitada para mercados poco desarrollados.

Código JEL: M41, O34, G32, C33

Palabras clave: Activos intangibles; Capital intelectual; Relevancia valorativa; Mercado de capitales; Argentina

\footnotetext{
*Autor para correspondencia

Correo electrónico: ceciliaficco@yahoo.com.ar (C.R. Ficco).

La revisión por pares es responsabilidad de la Universidad Nacional Autónoma de México. 


\begin{abstract}
The objective of this paper is to analyze the influence of the information on intangible assets and intellectual capital in the valuation of companies' shares in the argentine capital market, where there are no published works of this nature. The analysis is carried out applying a self-constructed model, based on Ohlson (1995). The data correspond to the period 2009-2018 and have been collected directly from original sources. They were obtained from the companies' consolidated annual financial statements and from the stock trading records of Buenos Aires Stock Market. The results reveal that identifiable intangible assets, human and structural capital are value relevant, contributing to increase the existing evidence regarding the role of intangibles in the external valuation of companies, which is particularly limited for under developed markets.
\end{abstract}

JEL Code: M41; O34, G32, C33

Keywords: Intangible assets; Intellectual capital; Value relevance; Capital market; Argentina

\title{
Introducción
}

Los intangibles tienen hoy un papel central en la generación de valor dentro de las empresas. Son los recursos estratégicos por excelencia y una de las fuentes principales de ventaja competitiva (Azofra, Ochoa, Prieto y Santidrián, 2017; Xu y Wang, 2018; Xu y Li, 2019), en un entorno que ha evolucionado hacia una economía basada en el conocimiento. La contabilidad presenta importantes deficiencias informativas en el actual contexto, en tanto, aun cuando los principales organismos emisores de normas han adoptado el enfoque de la utilidad de la información para la toma de decisiones (Scott, 2009), la postura dominante que han tenido con relación a los intangibles ha sido conservadora, siendo, en general, restrictiva en lo que respecta a su inclusión en los estados financieros.

Esto ha dado lugar a que solo unos pocos intangibles queden incluidos dentro del rubro "activos intangibles", y que una parte importante de ellos queden fuera de dichos estados, por no cumplir con los requisitos previstos para su reconocimiento contable. Estos elementos son habitualmente incluidos bajo la denominación de "capital intelectual", término que, en una de sus acepciones más usadas a partir de la conceptualización pionera de Edvinsson y Malone (1997) -y la que se adopta en esta investigación-, es empleado para hacer referencia a los elementos intangibles que pueden considerarse activos ocultos, en virtud de que no aparecen en los estados financieros de las empresas.

Las evidencias en torno a la importante brecha entre el valor en libros y el valor de mercado de las empresas cotizantes en las bolsas de valores, documentada inicialmente por Lev 
(2001) y reafirmada en investigaciones recientes (Kimouche y Rouabhi, 2016a y b), junto a las referidas a la debilidad en la relación entre variables de mercado e información contable (Lev y Zarowin, 1999; Hail, 2013; Badu y Appiah, 2018) ponen de manifiesto las limitaciones de los sistemas contables en vigor para brindar información útil para valoración externa de las empresas. Al mismo tiempo, revelan la existencia de otras fuentes de información, representativas de la creación de valor, que son tomadas en cuenta por los inversores y que impactan en el valor de mercado de las firmas, las que parecen estar directamente vinculadas a los intangibles y, en particular, al capital intelectual que no figura en los estados financieros.

Surge así la necesidad de examinar y contrastar en qué medida la información sobre intangibles, reconocidos y no reconocidos por la contabilidad, es relevante para la valoración de las empresas en los mercados de capitales, lo que resulta muy significativo en el marco de los cuestionamientos a la utilidad de la información contable para la toma de decisiones, en tanto, tal como señala el International Accounting Standards Board -IASB- (2018), en su marco conceptual, la relevancia es una de las características cualitativas fundamentales de la información financiera útil.

Este es el marco en el que se plantea la presente investigación, la cual tiene como objetivo analizar la influencia de la información sobre activos intangibles y capital intelectual en el valor que el mercado asigna a las empresas. Se trata de estudiar en qué medida las variables referidas a intangibles permiten explicar los precios observados en el mercado.

La investigación se realiza para el período temporal 2009-2018 y se contextualiza en el mercado de capitales (MCA), donde no se ha constatado la existencia de trabajos publicados de esta naturaleza, siendo además, un entorno idóneo para su estudio, en virtud de las especiales características que le otorgan su limitado desarrollo, la alta concentración del volumen negociado en pocas empresas y la baja permanencia de las mismas en los índices bursátiles (Dapena, 2012; Tolosa, 2013). El análisis se ha realizado aplicando un modelo de construcción propia, basado en el marco de valoración de Ohlson (1995). Los datos se recolectaron directamente de fuentes originales y se obtuvieron tanto de los estados financieros anuales consolidados presentados en la Bolsa de Comercio de Buenos Aires, como de los registros referidos a negociación de acciones en el Mercado de Valores de Buenos Aires.

El trabajo se ha estructurado en la forma que a continuación exponemos. En primer lugar, se presenta la revisión de la literatura, la que involucra los aspectos conceptuales y normativos más significativos referidos a los recursos intangibles que son objeto de este trabajo, una exhaustiva atención a la literatura empírica previa que ha examinado el papel de los intangibles para el mercado y la presentación de las teorías que dan sustento a este estudio. En la tercera sección del trabajo se expone el diseño de la investigación y, en la cuarta, los resultados empíricos y su discusión. El trabajo finaliza señalando sus principales conclusiones y contribuciones, sentando un precedente en cuanto al modelo empleado para examinar la 
relación entre intangibles y valores de mercado y a las variables incluidas en él, lo que puede resultar susceptible de posteriores réplicas.

\section{Revisión de la literatura}

\section{Marco conceptual y normativo}

Los recursos intangibles sobre los que se centra este estudio incluyen una variada gama de elementos que pueden clasificarse en dos grandes categorías: activos intangibles y capital intelectual.

La expresión "activos intangibles" (AI) es habitualmente utilizada para hacer "referencia exclusivamente a aquellas inversiones de naturaleza intangible que, de acuerdo con las normas contables, pueden ser reconocidas como activos y, consiguientemente, pueden ser reflejadas en el balance de la empresa" (Cañibano, Sánchez, García y Chaminade, 2002, p. 16). La contabilización de AI es un tema que viene siendo discutido por la literatura contable desde hace más de un siglo, sin que se haya llegado a un acuerdo respecto de los criterios para su reconocimiento y medición. No obstante, en general, tanto las normas contables argentinas (NCA) como las normas internacionales de información financiera (NIIF) del IASB ${ }^{1}$, requieren, para el reconocimiento de AI, la posibilidad de obtención de beneficios económicos futuros y la medición confiable, agregándose el requisito de identificabilidad en la NIC 38 (IASB, 2018).

La carencia de criterios generalmente aceptados de reconocimiento también genera una falta de acuerdo acerca de cuáles son las inversiones que pueden considerarse como AI. Sin embargo, pueden distinguirse dos categorías fundamentales de acuerdo al criterio de identificabilidad: llave de negocio ${ }^{2}$ y otros activos intangibles identificables, que son las que los cuerpos normativos referidos han diferenciado para regular su tratamiento contable.

En lo que respecta a estos últimos, tanto las NCA como las NIIF, regulan su reconocimiento y medición diferenciando, básicamente, entre los adquiridos y los generados internamente. Además, explicitan ciertos conceptos que no pueden ser reconocidos como activos, como es el caso de los desembolsos vinculados a la investigación u otros realizados para el desarrollo interno de marcas, listas de clientes y similares. La normativa argentina se diferencia por permitir la activación de los costos de organización y pre-operativos, situación no contemplada

\footnotetext{
${ }^{1}$ El Marco Conceptual para la Información Financiera, la NIC 38 y la NIIF 3 del IASB regulan la contabilización de AI. En Argentina rigen las Resoluciones Técnicas (RT) emitidas por la Federación Argentina de Consejos Profesionales de Ciencias Económicas (FACPCE, 2020), específicamente, las RT 16, 17, 18 y 21. La RT 26 fijó la obligatoriedad de aplicación de NIIF para todas las empresas cotizantes -excepto las financieras, de seguros, cooperativas y asociaciones civiles- para los ejercicios iniciados en 2012 , salvo para las de transporte y distribución de gas cuya vigencia se difirió a los ejercicios iniciados en 2013.

2 La expresión "llave de negocio" es la de uso común en Argentina. En España se utiliza el término "fondo de comercio", mientras que en otros países de Latinoamérica se lo refiere como "crédito mercantil". En el idioma inglés, se emplea la expresión goodwill, la que, en las NIIF, ha sido traducida al español, por el propio IASB, como "plusvalía".
} 
en la NIC 38 (IASB, 2018). Con relación a la llave de negocio, ambos cuerpos normativos admiten solo el reconocimiento de la que fuera adquirida en una combinación de negocios. Sin embargo, las NCA postulan que debe reconocerse un valor llave negativo cuando el precio pagado en la transacción resulte inferior al importe de los activos identificables netos adquiridos, mientras que las NIIF indican que esa diferencia se reconozca como ganancia (Ficco, 2019).

El término "capital intelectual" (CI), en cambio, representa un concepto mucho más amplio, en relación al cual aún no se ha llegado a una definición de aceptación generalizada. No obstante, sobre la base de las principales características que están presentes en la mayoría de los conceptos elaborados, podemos indicar que el CI refiere al conjunto de elementos intangibles relacionados entre sí, entre los que destaca el conocimiento disponible -nivel individual y organizativo-, que están en cierta medida ocultos, por no estar reflejados en los estados financieros elaborados en base a la normativa vigente y que permiten a la empresa funcionar, en combinación con los demás recursos, siendo fuente de ventaja competitiva y de creación de valor (Edvinsson y Malone, 1997; Ross, Ross, Dragonetti y Edvinsson, 2001; Ginesti, Caldarelli y Zampella, 2018).

El CI incluye elementos de diversa índole que pueden agruparse en una serie de categorías, siendo las correspondientes a capital humano, estructural y relacional, las que identifican la mayor parte de los autores (Edvinsson y Malone, 1997; Bontis, 1998; Ross, 2017; Sardo y Serrasqueiro, 2018; Tejedo y Araujo, 2018). El capital humano está dado por el stock de conocimientos individuales que reside en los empleados (Bontis, Chong y Richardson, 2000) e incluye tanto los saberes que poseen los mismos, como sus capacidades, experiencias y habilidades (Cañibano et al., 2002). Se trata de conocimiento tácito (Bontis, 1998), por lo que presenta dificultades para ser retenido por la empresa. No obstante, es la fuente fundamental de otros recursos estratégicos (Bontis, 1998; Ginesti et al., 2018) y de la creación de valor (Smriti y Das, 2018), por lo que se considera como el componente clave del CI (Tyskbo, 2019).

El capital estructural es la infraestructura que incorpora, forma y sostiene al capital humano (Edvinsson y Malone, 1997). Pero a la vez lo potencia, permitiendo optimizar su rendimiento y, con ello, el de la empresa en su conjunto (Bontis, 1998). Es, esencialmente, conocimiento que la organización ha podido internalizar (Ross et al., 2001), por lo que revela su aptitud para trasmitir y acumular material intelectual (Ochoa, Prieto y Santidrián, 2010). Incluye, de este modo, las rutinas organizativas que permiten la conversión del conocimiento individual en colectivo (Bollen, Vergauwen y Schnieders, 2005). Involucra una amplia variedad de componentes que pueden agruparse en dos grandes bloques (Ochoa et al., 2010; Smriti y Das, 2018): los elementos internos actualmente operativos en la organización, como la infraestructura, los procesos y la cultura empresarial y aquellos que aludirían a la capacidad de renovación de la empresa y a los resultados de la innovación. Entre estos últimos se incluyen las marcas, patentes, derechos de autor, etc. (Cañibano et al., 2002; Ginesti et al., 2018; Tyskbo, 2019). 
El capital relacional está fundamentalmente asociado al valor que generan las relaciones que la empresa entabla con su entorno, a través del intercambio de información y de productos a largo plazo con diferentes stakeholders, entre los que se incluyen: clientes, proveedores, socios aliados y accionistas (Ross et al., 2001; Forte, Matonti y Nicolò, 2019). Se trata pues de una dimensión externa a la organización, aunque en el valor de esas relaciones, como bien señala Sveiby (2018), influyen las acciones que desarrollan sus miembros, que son quienes las inician y mantienen. Más aún, algunos autores han remarcado la importancia del capital estructural como soporte para establecer y sostener las relaciones con stakeholders externos (Sardo y Serrasquiero, 2018).

\section{Revisión de la literatura empírica previa}

Es abundante la evidencia empírica sobre la relevancia de los intangibles para la valoración de las empresas por parte de los inversores. No obstante, en su mayoría, refiere a mercados de capitales con un grado de desarrollo significativo. Además, es posible identificar dos grandes líneas de trabajos claramente independientes, realizadas dentro de lo que Andriessen (2004) identifica como comunidades diferentes: la contable y la del CI. En la primera línea, se ha estudiado la relevancia valorativa de los intangibles reconocidos contablemente, mientras que en la segunda se ha examinado la de los distintos componentes del CI.

La idea de relevancia valorativa tiene diversas interpretaciones. No obstante, la más común, y la adoptada en el presente trabajo siguiendo a Barth, Beaver y Landsman (2001), es la que sostiene que una cifra contable (o no contable) es relevante para los inversores si posee una asociación significativa con los precios del mercado.

Las investigaciones realizadas por la comunidad contable revelan la existencia de una asociación significativa y positiva entre la cifra global correspondiente a los AI y el valor de mercado de los títulos (Choi, Kwon y Lobo, 2000; Iñiguez y López, 2005; Priotto, Quadro, Veteri y Werbin 2011). No obstante, en lo que respecta a las distintas categorías de AI, las evidencias son sólidas en lo atinente a la relevancia valorativa de los identificables, pero no resultan del todo concluyentes para la llave de negocio.

Así, Ritter y Wells (2006), Dahmash, Durand y Watson (2009), Oliveira, Rodrigues y Craig (2010), Kimouche y Rouabhi (2016a), Da Silva, Rodrigues y Klann (2017) y Omarjee, Yasseen y Mohamed (2019) constatan la relevancia valorativa de las dos categorías. Ocak y Findik (2019), en un estudio realizado sobre activos intangibles identificables, obtienen evidencia que muestra su influencia positiva en el valor de mercado de las firmas. Kimouche y Rouabhi (2016b) también obtienen hallazgos que revelan una asociación positiva entre dichos activos y los precios de mercado, pero encuentran que esa asociación es negativa para la llave de negocio. Infante y Ferrer (2017), en cambio, detectan que la llave de negocio no es una variable considerada por los inversores. 
Estos resultados se explican, en gran parte, por la particular naturaleza de la llave de negocio, que no resulta directamente observable después de su adquisición. Por ello, la cifra informada en los estados financieros solo resulta representativa del valor del intangible en el momento de la combinación de empresas (Giner y Pardo, 2007), lo que da lugar a que la normativa contable aplicable a la medición de este activo, en los ejercicios posteriores a su reconocimiento inicial, genere una influencia muy significativa en su valoración a nivel del mercado de capitales, tal como queda patente en Bepari y Mollik (2017), Wahyuni, Dewantoro y Avianti (2018), Omarjee et al. (2019) y Kwon y Wang (2020).

En la línea de investigación sobre CI, se ha examinado la relación entre sus distintas dimensiones -humana, estructural y relacional- y el valor de mercado de las empresas, lo que ha dado lugar, en determinados casos, a resultados mixtos. Dado que estas investigaciones se concentran sobre elementos que no figuran en los estados financieros y que, además, son de difícil medición, en general, han empleado proxies para medirlos, utilizando para ello tanto información financiera como no financiera.

En lo que respecta al capital humano, la literatura previa aporta evidencias sólidas acerca de su impacto positivo en la formación de precios. Así, Yu y Zhang (2008), que lo miden con una proxy referida a la productividad de los empleados, confirman su relevancia valorativa, al igual que García, Rodríguez y García (2018), que usan como proxy a la inversión acumulada en capacitación de empleados. Wang (2008) y Liu, Tseng y Yen (2009) obtienen resultados en igual sentido, al encontrar que algunas de las proxies que emplean para medir el capital humano se asocian positivamente con los precios de mercado. Los estudios de Swartz, Swartz y Firer (2006), Nimtrakoom (2015), Suherman (2017), Villegas, Hernández y Salazar (2017), Sardo y Serrasqueiro (2017) y Bayraktaroglu, Calisir y Baskak (2019) encuentran una asociación significativa y positiva entre el indicador de eficiencia en el uso del capital humano -calculado a partir del Value Added Intellectual Coefficient -VAIC- (Pulic, 2000)- y los valores de las empresas en el mercado. Ferraro y Veltri (2011), si bien no encuentran evidencias de una influencia directa del capital humano en los precios, logran demostrar que posee efecto indirecto cuando se lo examina en su interacción con el capital estructural, lo que resulta consistente con los resultados que obtienen Cabrita y Bontis (2008), Özer y Çam (2017) y Barbosa, Coelho y Weersma (2019). Aunque resultan minoritarios, ciertos estudios han obtenido evidencia de una asociación significativa y negativa entre el capital humano y los precios del mercado. Entre ellos, los de Smriti y Das (2018) y los de Forte et al. (2019), quienes, en línea con lo anterior, han interpretado que tales resultados derivan de la evaluación de la relevancia de los componentes del CI de manera individual, cuando resulta fundamental el efecto combinado que produce su interacción.

En cuanto al estudio de la relación entre el capital estructural y el valor de mercado de las empresas, algunos trabajos, teniendo en cuenta la variedad de componentes que integran 
esta dimensión, han examinado separadamente el capital organizacional o de procesos y el capital de innovación. En esta línea, Wang (2008), empleando proxies basadas en los gastos administrativos y de comercialización para medir el capital de procesos y los gastos en I+D para medir el de innovación, obtienen resultados que apoyan la relevancia valorativa de ambas dimensiones. Similares resultados obtienen Liu et al. (2009), aunque usando diferentes medidas para cada una de ellas. Sharma (2018), en cambio, encuentra una asociación significativa, pero negativa, entre el capital de innovación y los precios de mercado, al igual que Ferraro y Veltri (2011) entre capital de procesos y precios. En este último trabajo, además, y en forma coincidente con Yu y Zhang (2008), se obtienen evidencias de que capital de innovación no es tomado en cuenta en la valoración de las empresas. Por su parte, Veltri y Silvestri (2011), Nimtrakoom (2015), Suherman (2017), Sardo y Serrasqueiro (2017) y Smriti y Das (2018) encuentran una asociación positiva y significativa entre el indicador que mide la eficiencia en el uso del capital estructural -calculado sobre la base del VAIC- y los precios del mercado. En cambio, Swartz et al. (2006), Sharma (2018), Bayraktaroglu et al. (2019) y Soetanto y Liem (2019) obtienen evidencias de que dicho indicador no es significativo para la formación de precios, mientras que Forte et al. (2019) encuentran que el mismo está relacionado de manera significativa, pero negativa, con los valores de mercado de las firmas.

En lo atinente al capital relacional, los estudios previos no han logrado demostrar de manera contundente su relevancia valorativa. Así, Yu y Zhang (2008) obtienen resultados que muestran que el gasto en publicidad es un generador de valor, pero detectan que el volumen de ventas no influye en la formación de precios. En el estudio de Wang (2008), en cambio, el gasto en publicidad presenta una asociación negativa con los precios, pero la variable que mide la tasa de crecimiento de las ventas se asocia positivamente con los mismos. Ferraro y Veltri (2011), usando a las ventas como proxy del capital relacional y Sharma (2018), tomando como proxy al gasto en publicidad, concluyen que esta dimensión del CI es valorada positivamente por los inversores. García et al. (2018) también constatan la relevancia valorativa del capital relacional, al cual miden a través de la inversión acumulada en publicidad. Y Liu et al. (2009), Nimtrakoom (2015), Suherman (2017) y Soetanto y Liem (2019) obtienen resultados que muestran que el capital relacional no tiene impacto en el valor corporativo que asigna el mercado.

De este modo, la revisión de la literatura deja en claro la diversidad de resultados obtenidos en relación con el vínculo entre intangibles y valor de mercado de las empresas, a la vez que confirma que las investigaciones previas han estudiado, separadamente, la relevancia valorativa de los AI y del CI. Esta situación nos advierte sobre la necesidad de aportar evidencia sobre una relación cuyo abordaje se encuentra aún inconcluso, así como de brindar una visión completa sobre los determinantes intangibles del valor de las empresas que permita, además, aumentar la limitada evidencia existente para mercados poco desarrollados. Con este fin, se desarrolla la investigación empírica que presentamos seguidamente. 
Teorías que explican la divulgación de información y el papel asignado a la misma

Los estudios de relevancia valorativa de intangibles se pueden apoyar en distintas teorías que se han empleado para explicar la divulgación de información por parte de las empresas y el papel asignado a la misma, como la teoría de la agencia, la de los stakeholders y la de la señal.

La teoría de la agencia (Jensen y Meckling, 1976) se centra, principalmente, en la relación que se da entre los accionistas de la empresa (principales) y los directivos (agentes), la cual se caracteriza por la divergencia de intereses entre las partes y por una distribución asimétrica de la información, que puede originar comportamientos oportunistas. De acuerdo a esta teoría, la divulgación de información es un medio para reducir esa asimetría informativa y para mitigar los costos de agencia que de ella se derivan (Larrán y García, 2004; Rieg y Vanini, 2015). En particular, la información sobre intangibles brinda a los accionistas un mayor conocimiento sobre la empresa (Ellis y Seng, 2015) y, de ese modo, les sirve para tomar decisiones más eficientes, al aportar información sobre los principales generadores de valor (Tejedo, Ferraz y Emmendoerfer, 2017).

Para la teoría de los stakeholders, enunciada por Freeman (1984), la empresa se concibe como un conjunto de stakeholders que interactúan entre sí de manera constante y dinámica, siendo las relaciones que la empresa mantiene con ellos la clave para su supervivencia y crecimiento. De este modo, la empresa debe cumplir con las expectativas de todos sus participantes, por lo que tiene la responsabilidad de darles cuentas de su gestión con el fin de ayudarlos a tomar decisiones apropiadas (Ríos, Torres, Tirado y Carbonell, 2009). En este marco, la revelación de información es considerada como parte del diálogo que permite negociar la relación entre la empresa y sus stakeholders (Ochoa, Prieto y Santidrián, 2012). Específicamente, la información sobre intangibles es un elemento para obtener el apoyo y la aprobación de los mismos (Whiting y Miller, 2008), en la medida que, al referir a recursos estratégicos, permite aumentar el valor percibido que tienen de la empresa (Tejedo y Alfaro, 2014).

La teoría de la señal tiene importantes relaciones con las anteriores. Desde esta perspectiva, la revelación de información por parte de la empresa constituye un mecanismo para señalar al mercado su mejor posición relativa frente a otras empresas (Ross, 1979), lo que también contribuye a disminuir las asimetrías de información (Kimouche y Rouabhi, 2016b) y, de este modo, a la mejora en la toma de decisiones de los inversores actuales y potenciales y de otros stakeholders. En particular, la información sobre intangibles permite a las empresas señalar su superior calidad, por tratarse de los atributos corporativos más significativos para la creación de riqueza (Whiting y Miller,2008; Tejedo et al., 2017). 


\section{Diseño de la investigación}

Con el fin de atender al objetivo planteado en la introducción, construimos un modelo basado en Ohlson (1995) que permite contrastar, para la totalidad de empresas que integran la muestra, la asociación entre las variables referidas a intangibles y el valor que el mercado les asigna.

Para estudiar la diversidad de intangibles presentes en las empresas hemos contemplado tanto los reconocidos como los no reconocidos contablemente. Así, el modelo incluye, como variables independientes, a las dos categorías fundamentales de activos intangibles ( $A I)$ : llave de negocio $(L L N)$ y otros activos intangibles $(O A I)$, obtenidas de los estados financieros de las empresas, y a las tres dimensiones de mayor consenso para agrupar a los componentes del capital intelectual $(C I)$ : capital humano $(C H)$, estructural $(C E)$ y relacional $(C R)$, para cuya medición se utilizan proxies basadas en información contable. Como variable dependiente, representativa de los valores de mercado, hemos tomado los precios de las acciones $(P)$. La Figura 1 esquematiza el modelo de trabajo propuesto y las variables que se someten a estudio, lo que permite dar marco a las hipótesis de la investigación.

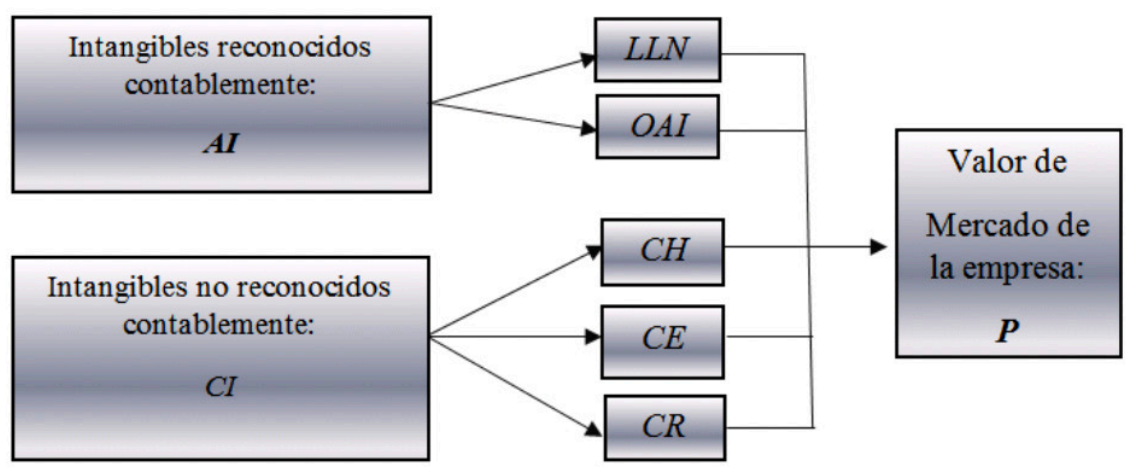

Figura 1. Modelo de trabajo y variables sometidas a estudio.

Fuente: Elaboración propia.

\section{Desarrollo de las hipótesis de la investigación}

La revisión de la literatura empírica previa que ha examinado la relevancia valorativa de los AI y del CI proporciona evidencia de la asociación entre la información referida a estos elementos y la valoración de las empresas en el mercado (Ritter y Wells, 2006; Wang, 2008; Dahmash et al., 2009; Oliveira et al., 2010; Ferraro y Veltri, 2011; Nimtrakoom, 2015; Ki- 
mouche y Rouabhi, 2016a y b; Da Silva et al., 2017; Sardo y Serrasqueiro, 2017; García et al., 2018; Sharma, 2018; Ocak y Findik, 2019; entre otros).

Esta evidencia empírica, junto a las explicaciones aportadas por las teorías de la agencia, de los stakeholders y de la señal con relación a la divulgación de información por parte de las empresas y al papel asignado a la misma, dan sustento a la idea de que los distintos tipos de intangibles, reconocidos y no reconocidos contablemente, pueden tener efectos valorativos en el MCA. En efecto, es de esperar que los inversores consideren la información referida a los mismos en sus decisiones de compra y venta de acciones, lo que producirá cambios sobre sus precios. Concretamente, se espera que presenten una asociación positiva con el valor de mercado de las empresas, por ser, todos ellos, representativos de la creación de valor. Por lo tanto, las hipótesis de la investigación quedan formuladas de la siguiente forma:

H1a: La $L L N$ reconocida en los estados financieros de las empresas cotizantes en el MCA tiene relevancia para la valoración de sus acciones en dicho mercado.

H1b: Los $O A I$ reconocidos en los estados financieros de las empresas cotizantes en el MCA tienen relevancia para la valoración de sus acciones en dicho mercado.

H2a: El $\mathrm{CH}$ de las empresas cotizantes en el MCA tiene relevancia para la valoración de sus acciones en dicho mercado.

H2b: El CE de las empresas cotizantes en el MCA tiene relevancia para la valoración de sus acciones en dicho mercado.

H2c: El $C R$ de las empresas cotizantes en el MCA tiene relevancia para la valoración de sus acciones en dicho mercado.

\section{Construcción del modelo y definición de variables}

La construcción del modelo que permite contrastar las hipótesis formuladas se basa en el desarrollado por Ohlson (1995), por ser un modelo de valoración de la empresa que centra la atención en los "determinantes del valor" (AECA, 2006, p. 10). Específicamente, formaliza el vínculo entre el valor de la firma y las variables contables fundamentales: patrimonio neto y resultado, incluyendo, además, una variable que representa “otra información” aún no reflejada en los estados financieros pero que se espera tendrá impacto en los resultados futuros. Se trata pues de un modelo de valoración completo, que aporta una base metodológica sólida para comprender la relación entre el valor de la empresa en el mercado y las variables contables y no contables, lo que permite evaluar si son relevantes para los inversores.

En concreto, se parte de una versión del modelo de Ohlson (1995) ampliamente usada por la investigación previa, que se especifica del siguiente modo: 


$$
P_{i t}=\beta_{0}+\beta_{1} P N_{i t}+\beta_{2} R D O_{i t}+\beta_{3} v_{i t}+\varepsilon_{i t}
$$

donde $P_{i t}$ es el valor de mercado de las acciones de la empresa $i$ en el momento $t, P N_{i t}$ es el valor contable del patrimonio neto de la empresa $i$ en el momento $t, R D O_{i t}$ es el resultado contable de la empresa $i$ para el período $t$ y $v_{i t}$ es "otra información" que influye en el valor de la empresa $i$ pero que no está contenida en sus estados financieros en el momento $t$.

A fin de incorporar las variables relativas a los distintos tipos de intangibles, se realizan modificaciones sobre la expresión (1), en dos sentidos. Por un lado, se descompone el $P N$ en dos componentes: activos intangibles $(A I)$ y valor contable de los elementos tangibles netos $(T G P N)$, siguiendo el planteo original de Ely y Waymire (1999). A su vez, los $A I$ se consideran desglosados en sus dos categorías fundamentales: $L L N$ y $O A I$. Por otro lado, se identifica a la variable "otra información" $(v)$ con la referida al capital intelectual $(C I)$ en sus distintas dimensiones ( $\mathrm{CH}, \mathrm{CE}$ y $\mathrm{CR}$ ), en virtud de que los aspectos humanos, organizativos y relacionales de la empresa, aunque no tienen reflejo en los estados financieros, poseen una importante capacidad para producir efectos sobre su desempeño futuro. Esta idea se fundamenta en distintas posiciones teóricas que sustentan el propio concepto de CI (Wernerfelt, 1984; Barney, 1991) y ha sido considerada también en algunos estudios empíricos previos (Wang, 2008; Yu y Zhang, 2008; Liu et al., 2009; Ferraro y Veltri, 2011 y Veltri y Silvestri, 2011).

Para medir los distintos componentes del CI se emplean proxies basadas en medidas contables, que se encuadran dentro de los métodos financieros de medición del CI (Andriessen, 2004). Además, se ha adoptado el enfoque basado en la inversión (Goebel, 2015; Forte, Tucker, Matonti y Nicolò, 2017), en tanto la mayoría de las proxies seleccionadas se basan en gastos que pueden ser considerados como inversiones con capacidad para generar beneficios económicos futuros vinculados al conocimiento existente dentro de la organización. Se consideraron dos proxies para cada dimensión, siguiendo a Sveiby (1997), quien sugiere usar pocos indicadores para tener un sistema de medición simple y representativo.

Las proxies seleccionadas figuran en la Tabla 1, junto con los estudios previos que sustentan tal selección. 


\section{C.R. Ficco, et al. / Contaduría y Administración 66 (3), 1-26}

http://dx.doi.org/10.22201/fca.24488410e.2021.2558

Tabla 1

Variables proxy seleccionadas para las distintas dimensiones del CI

\begin{tabular}{|c|c|c|}
\hline $\begin{array}{c}\text { Dimensión } \\
\text { del CI }\end{array}$ & Variables proxy seleccionadas & Estudios previos en los que se sustentan \\
\hline $\begin{array}{c}\text { Capital } \\
\text { humano } \\
\qquad(\mathrm{CH})\end{array}$ & $\begin{array}{c}\text { Gastos de Personal } \\
\text { (GP) } \\
\text { Ventas / Gastos de Personal } \\
(\text { VTAS/GP) }\end{array}$ & $\begin{array}{l}\text { Swartz et al. (2006), Ferraro y Veltri (2011), Veltri y Silvestri (2011), } \\
\text { Goebel (2015), Nimtrakoom (2015), Özer y Çam (2017), Suherman } \\
\text { (2017), Villegas et al. (2017), Sharma (2018), García et al. (2018), } \\
\text { Ginesti et al. (2018), Smriti y Das (2018) y Forte et al. (2019) } \\
\text { Swartz et al. (2006), Wang (2008), Yu y Zhang (2008), Liu et al. (2009), } \\
\text { Veltri y Silvestri (2011), Nimtrakoom (2015), Suherman (2017) y } \\
\text { Sharma (2018) }\end{array}$ \\
\hline $\begin{array}{c}\text { Capital } \\
\text { estructural } \\
\quad(C E)\end{array}$ & $\begin{array}{c}\text { Gastos administrativos } \\
\text { y de comercialización } \\
\text { (GAyC) } \\
\text { Gastos administrativos y de } \\
\text { comercialización / Ventas } \\
\text { (GAyC/VTAS) }\end{array}$ & $\begin{array}{l}\text { Lev, Radhakrishnan y Zhang (2009), Ferraro y Veltri (2011), Goebel } \\
\text { (2015), Lev, Radhakrishnan, Evans (2016) y Mačerinskienė y } \\
\text { Survilaitė (2019) } \\
\text { Wang (2008) y Yu, Wang y Chang (2009) }\end{array}$ \\
\hline $\begin{array}{l}\text { Capital } \\
\text { relacional } \\
\qquad(C R)\end{array}$ & $\begin{array}{c}\text { Ventas } \\
\text { (VTAS) } \\
\text { Gasto de Publicidad } \\
\text { (PUB) }\end{array}$ & $\begin{array}{l}\text { Yu y Zhang (2008), Wang (2008), Liuetal. (2009), Ferraro y Veltri (2011) } \\
\text { y Iazzolino, Chiappetta y Chiappetta (2018) } \\
\text { Yu y Zhang (2008); Wang (2008); Liu et al. (2009), Yu et al. (2009), } \\
\text { Nimtrakoon (2015), Suherman (2017), Sharma (2018), García et al. } \\
\text { (2018), Xu y Wang (2018), Soetanto y Liem (2019) y Xu y Li (2019) }\end{array}$ \\
\hline
\end{tabular}

Fuente: elaboración propia.

De acuerdo a las consideraciones anteriores, se modifica la expresión (1) para plantear el modelo esquematizado en la Figura 1, tal como se indica seguidamente:

$$
\begin{gathered}
P_{i t+3}=\beta_{0}+\beta_{1} T_{G P N_{i t}}+\beta_{2} R D O_{i t}+\beta_{3} L L N_{i t}+\beta_{4} O A I_{i t}+\beta_{5} G P_{i t}+\beta_{6} V T A S / G P_{i t}+\beta_{7} G A y C_{i t} \\
+\beta_{8} G A y C / V T A S_{i t}+\beta_{9} V T A S_{i t}+\beta_{10} P U B_{i t}+\varepsilon_{i t}
\end{gathered}
$$

donde las variables se definen de acuerdo a lo indicado en la Tabla 2.

Dos cuestiones merecen destacarse en lo relativo a la definición de las variables. En primer lugar, que la forma de cálculo de los precios de las acciones contempla los tiempos que demora el mercado en incorporar la información contable a las decisiones. En segundo lugar, los valores de las variables explicativas se emplean deflactados por el número de acciones, siguiendo la postura de Barth y Clinch (2009), quienes lo proponen como una forma efectiva para mitigar los sesgos que produce el efecto escala en la estimación de modelos econométricos. 
Tabla 2

Definición de variables

\begin{tabular}{ll}
\hline Simbología & \\
\hline$P_{i t+3}$ & Precio promedio por acción de la empresa i en el tercer mes siguiente al cierre del ejercicio t \\
$T G P N_{i t}$ & Patrimonio neto tangible (por acción) de la empresa i al cierre del ejercicio t \\
$R D O_{i t}$ & Resultado neto después de impuestos (por acción) de la empresa i para el ejercicio t \\
$L L N_{i t}$ & Llave de negocio (por acción) informada en los estados financieros de la empresa i al cierre del \\
& ejercicio t (neta de las depreciaciones ${ }^{3}$ acumuladas y de las pérdidas por desvalorización) \\
& Otros activos intangibles (por acción) informados en los estados financieros de la empresa i al \\
& cierre del ejercicio t (netos de las depreciaciones acumuladas y de las pérdidas por desvalorización) \\
$G P_{i t}$ & Gastos de personal (por acción) de la empresa i para el ejercicio t \\
$V T A S / G P_{i t}$ & (Ventas / Gastos de personal) de la empresa i para el ejercicio t \\
$G A y C_{i t}$ & Gastos administrativos y de comercialización (por acción) de la empresa i para el ejercicio t (netos \\
& de gastos de personal y de publicidad) \\
$G A y C / V T A S_{i t}$ & (Gastos administrativos y de comercialización* / Ventas) de la empresa i para el ejercicio t \\
& * netos de gastos de personal y de publicidad \\
$V T A S_{i t}$ & Ingresos netos por ventas (por acción) de la empresa i para el ejercicio t \\
$P U B_{i t}$ & Gastos de publicidad (por acción) de la empresa i para el ejercicio t \\
\hline
\end{tabular}

Fuente: elaboración propia.

La estructura de los datos disponibles, determinada por variables que se miden para cada empresa en distintos años, impone la necesidad de emplear un modelo para datos correlacionados. Por ello, desde el punto de vista econométrico, el modelo (2) se utiliza bajo la especificación de un modelo lineal para datos de panel. Más concretamente, para captar la heterogeneidad no observable entre sujetos se ha elegido una especificación de efectos fijos, en virtud de que, como señala De Jager (2008), es la más apropiada para la mayor parte de las investigaciones contables. Asimismo, para resolver los problemas de dispersión de los valores de la variable dependiente $(P)$, se trabaja con una transformación de la misma, por lo que la variable respuesta es el logaritmo natural del precio de la acción (InP). Así, el modelo a estimar es el siguiente:

$$
\begin{gathered}
\ln P_{i t+3}=\beta_{0}+\beta_{1} T G P N_{i t}+\beta_{2} R D O_{i t}+\beta_{3} L L N_{i t}+\beta_{4} O A I_{i t}+\beta_{5} G P_{i t}+\beta_{6} V T A S / G P_{i t}+\beta_{7} G A y C_{i t} \\
+\beta_{8} G A y C / V T A S_{i t}+\beta_{9} V T A S_{i t}+\beta_{10} P U B_{i t}+\mu_{i}+\varepsilon_{i t}
\end{gathered}
$$

donde $\mu_{i}$ es una variable aleatoria que permite captar la heterogeneidad individual no observable y las demás variables se definen de acuerdo a lo indicado en la Tabla 2.

\footnotetext{
${ }^{3}$ Se emplea el término "depreciación”, siguiendo la terminología usada por las normas contables argentinas.
} 


\section{C.R. Ficco, et al. / Contaduría y Administración 66 (3), 1-26}

http://dx.doi.org/10.22201/fca.24488410e.2021.2558

Para la estimación del modelo (3) se utiliza el estimador dentro (within estimator), que es la estrategia más apropiada para los modelos de efectos fijos, por permitir lograr estimaciones consistentes de los parámetros $\beta$ (Cameron y Trivedi, 2005). Adicionalmente, se han realizado estimaciones robustas basadas en White (1980), por ser esta una metodología de amplia aceptación como solución a la heterocedasticidad en los errores de la regresión. Sirve, también, para dar respuesta a la autocorrelación serial, siempre que se cumpla la independencia transversal (Baltagi, 2013; Díaz y Vargas, 2017).

\section{Espacio temporal y muestra}

La investigación se realiza sobre una muestra de empresas cotizantes en el MCA, pertenecientes a distintos sectores de actividad. El período elegido es el comprendido entre 2009 y 2018, ambos incluidos, lo que proporciona un rango temporal amplio para el análisis ${ }^{4}$.

Dado que el MCA se caracteriza por una baja permanencia de las empresas en los índices bursátiles que produce una situación de cambios en el panel de cotizantes, para seleccionar a las empresas que forman parte de la muestra se consideraron solo aquellas que se han mantenido en cotización de manera continua durante el período 2009-2018, identificándose 52 empresas en esta situación.

Para este conjunto se procedió a recolectar la información contable y del mercado necesaria para la investigación, lo que permitió obtener un total de 520 observaciones anuales. De este total, se eliminaron las observaciones para las cuales las variables patrimonio neto y/o resultado contable asumen valores negativos, por no ser consistentes con el marco de valoración de Ohlson (1995). La existencia de 118 observaciones con esa condición hace que la muestra total se reduzca a 402 observaciones. Se eliminaron también las observaciones con valores extremadamente atípicos, considerando como tales a aquellos que se alejan del valor de la media más de 5 veces la desviación típica en al menos dos de las variables explicativas del estudio ${ }^{5}$. Esto condujo a eliminar un total de 49 observaciones, lo que deja una muestra final conformada por 353 observaciones correspondientes a 46 empresas.

\footnotetext{
${ }^{4}$ En 2008 algunos hechos macroeconómicos impactaron significativamente en el MCA. Por ello, para evitar las distorsiones que los datos correspondientes a dicho año podrían generar en el presente estudio, el espacio temporal elegido se inicia en 2009. Asimismo, finaliza en 2018, que se corresponde con el último ejercicio económico para el cual se encuentran disponibles -al momento de realizar esta investigación- los estados financieros anuales consolidados de las empresas estudiadas.

${ }^{5}$ Normalmente se consideran como valores extremos a aquellos datos fuera del intervalo $\left(x^{-}-3 \sigma, x^{-}+3 \sigma\right)$, , o a lo sumo, fuera del intervalo $\left(x^{-}-4 \sigma, x^{-}+4 \sigma\right)$, donde es la media de los valores de la variable y es la desviación típica. Adicionalmente, se analizaron tres medidas de uso habitual para determinar valores atípicos e influyentes en un modelo de regresión: los residuos estudentizados y los estadísticos DFFITS y COVRATIO. El 70\% de las observaciones eliminadas coincide con las que estas medidas señalan como atípicas o potencialmente influyentes, lo que apoya el procedimiento de eliminación usado.
} 


\section{Resultados empíricos y discusión}

En este epígrafe se presenta el análisis descriptivo de la muestra, los resultados obtenidos a partir de la estimación del modelo (3) y su discusión.

Los estadísticos descriptivos de la muestra se resumen en la Tabla 3. En ella se puede observar que todas las variables explicativas presentan coeficientes de variación altos, lo que indica que se trata de variables con gran dispersión. Se aprecia también que la transformación de $P$ a la forma logarítmica $(\operatorname{In} P)$ ha permitido reducir la dispersión de los datos referidos a precios.

Tabla 3

Principales estadísticos descriptivos de la muestra

\begin{tabular}{ccccccc}
\hline Variable & Media & Desviación típica & Coef. de variación & Mediana & Máximo & Mínimo \\
\hline$P^{*}$ & 21.97 & 37.54 & 1.71 & 8.00 & 440.60 & 0.19 \\
InP & 2.20 & 1.35 & 0.61 & 2.08 & 6.09 & -1.66 \\
$T G P N^{*}$ & 6.99 & 9.25 & 1.32 & 3.60 & 63.09 & -7.49 \\
$R D O^{*}$ & 1.86 & 2.84 & 1.53 & 0.71 & 15.96 & 0.00 \\
LLN* & 0.04 & 0.44 & 10.13 & 0.00 & 7.55 & -1.48 \\
$O A I^{*}$ & 0.38 & 1.13 & 2.99 & 0.02 & 8.54 & 0.00 \\
$G P^{*}$ & 2.57 & 3.01 & 1.17 & 1.49 & 21.23 & 0.01 \\
$V T A S / G P^{*}$ & 8.46 & 14.93 & 1.76 & 5.42 & 173.20 & 0.00 \\
$G A y C^{*}$ & 1.93 & 3.28 & 1.70 & 0.70 & 21.40 & 0.00 \\
$G A y C / V T A S^{*}$ & 0.14 & 0.21 & 1.54 & 0.11 & 2.99 & 0.00 \\
VTAS* & 17.62 & 32.86 & 1.86 & 7.83 & 489.02 & 0.00 \\
PUB* & 0.14 & 0.35 & 2.44 & 0.01 & 2.82 & 0.00 \\
\hline
\end{tabular}

* Valores expresados en moneda argentina (pesos) por acción.

Fuente: Elaboración propia.

La Tabla 4 recoge los resultados obtenidos en la estimación del modelo (3) con datos correspondientes al período 2009-2018. Se observa que el coeficiente de correlación intraclásica $(r h o$ ) indica que el $52.92 \%$ de la variabilidad total se debe a la variabilidad entre las empresas, siendo esta heterogeneidad un indicador de que es apropiado usar efectos fijos. El test de máxima verosimilitud para la redundancia de efectos fijos arroja p-valores $<0,01$ (Test F y $C h i^{2}$ ), revelando que los efectos fijos de las empresas son diferentes con un $99 \%$ de confianza al menos, lo que también da sustento al uso de este modelo. El coeficiente de determinación ( $\mathrm{R}^{2}$ within) es mediano y semejante al de otros estudios que emplean modelos de panel de efectos fijos (Oliveira et al., 2010; Ferraro y Veltri, 2011; Wahyuni et al., 2018).

Los coeficientes y p-valores expuestos en la Tabla 4 revelan que las variables $R D O$, 
$O A I, G P, V T A S / G P, G A y C$ y GAyC/VTAS son estadísticamente significativas y positivas con un nivel de significación del 10\%, mientras que TGPN, LLN, VTAS y PUB no lo son. Estos resultados indican que los activos intangibles identificables $(O A I)$ proporcionan información relevante para los inversores, mientras que la llave de negocio $(L L N)$ no es significativa en el proceso de formación de precios. Respecto del CI, se evidencia que tanto el capital humano como el estructural poseen relevancia valorativa, ya que las dos proxies del capital humano (GP Y VTAS/GP) y las dos proxies del capital estructural (GAyC y GAyC/VTAS) son significativas y positivas. En cambio, ninguna de las proxies del capital relacional (VTAS y PUB) son significativas, lo que pone de manifiesto que esta dimensión no tiene impacto en el valor corporativo que asigna el mercado. De este modo, hemos obtenido evidencias en favor de las hipótesis $\mathrm{H} 1 \mathrm{~b}, \mathrm{H} 2 \mathrm{a}$ y $\mathrm{H} 2 \mathrm{~b}$, mientras que los resultados no apoyan los planteos realizados en las hipótesis H1a y H2c.

Tabla 4

Relevancia valorativa de los activos intangibles y del capital intelectual

\begin{tabular}{|c|c|c|c|c|}
\hline Variables explicativas & Coeficiente & Error estándar & Estadístico t & p-valor \\
\hline$T G P N$ & -0.0078 & 0.0205 & -0.38 & 0.706 \\
\hline$R D O$ & $0.1715^{* *}$ & 0.0701 & 2.45 & 0.018 \\
\hline$L L N$ & -0.0530 & 0.1092 & -0.49 & 0.629 \\
\hline$O A I$ & $0.1059 * *$ & 0.0487 & 2.18 & 0.035 \\
\hline$G P$ & $0.1750 * * *$ & 0.0522 & 3.35 & 0.002 \\
\hline$V T A S / G P$ & $0.0054^{*}$ & 0.0027 & 1.97 & 0.055 \\
\hline$G A y C$ & $0.0510^{*}$ & 0.0279 & 1.83 & 0.074 \\
\hline GAyC/VTAS & $0.2722 * * *$ & 0.0794 & 3.43 & 0.001 \\
\hline VTAS & -0.0019 & 0.0013 & -1.53 & 0.133 \\
\hline$P U B$ & -0.2164 & 0.2726 & -0.79 & 0.431 \\
\hline Constante & $1.3297 * * *$ & 0.0938 & 14.17 & 0.000 \\
\hline $\mathrm{N}^{\circ}$ observaciones & 353 & & & \\
\hline $\mathrm{R}^{2}$ within & 0.4680 & & & \\
\hline Correlación intraclásica (rho) & 0.5292 & & & \\
\hline Test F & $5.78 * * *$ & & & \\
\hline$C h i^{2}$ & $222.14 * * *$ & & & \\
\hline
\end{tabular}

Nota: Se ha utilizado el estimador dentro (within estimator) y se realizó estimación robusta basada en la corrección de White $(1980)^{6}$.

Los símbolos ***,** y* denotan que la variable es significativa al 1\%,5\% o $10 \%$, respectivamente. Las variables se definen de acuerdo a lo indicado en la Tabla 2.

Fuente: Elaboración propia.

${ }^{6}$ Como paso preventivo, se ha examinado la posibilidad de multicolinealidad entre las variables explicativas incluidas en el modelo (3), a través de los factores inflacionarios de la varianza (FIV). Siguiendo a Gujarti y Porter (2010), no hay indicios de multicolinealidad, en tanto los FIV resultaron todos menores a 10. 
Los resultados sugieren que, a pesar de los problemas que plantea el reconocimiento y la medición de los activos intangibles identificables, los inversores los perciben como activos legítimos, considerando que proporcionan información sobre los futuros beneficios de las empresas y que contribuyen a los mismos. También revelan que el MCA comprende la importancia del capital humano, como componente primario y clave del CI, y así también, la trascendencia del capital estructural como sostén y elemento potenciador del primero, ya que, como sostienen Nonaka y Takeuchi (1995), la interacción de ambos es el motor del proceso completo de creación de conocimiento.

Estos resultados contribuyen a las teorías de la agencia, de los stakeholders y de la señal que sustentan este estudio, ya que, al revelar que la información referida a estos elementos intangibles tiene un impacto favorable en los precios de las acciones, aportan evidencia de que los inversores la valoran como un instrumento para obtener un mayor conocimiento de la empresa y reducir las asimetrías de información que existen con relación a sus administradores; como parte del diálogo con los stakeholders que permite dar cuentas de la gestión de la empresa a todos sus partícipes y revelar el valor creado para todos ellos y, así también, como una señal de una mejor posición relativa de la empresa frente a las demás.

Los elementos intangibles que resultan relevantes para los inversores participantes en el MCA son coincidentes con aquellos sobre los cuales la literatura previa ha aportado las evidencias más sólidas. Así, nuestros hallazgos reconfirman los de Ritter y Wells (2006), Oliveira et al. (2010), Kimouche y Rouabhi (2016a y b), Da Silva et al. (2017), Omarjee et al. (2019) y Ocak y Findik (2019), que comprueban que los inversores tienen en cuenta a los activos intangibles identificables en la fijación del precio de las acciones, y los de Wang (2008), Liu et al. (2009), Nimtrakoom (2015), Suherman (2017) y Sardo y Serrasquiero (2017), que encuentran evidencias que apoyan la relevancia valorativa tanto del capital humano como del estructural.

La llave de negocio no resultó una variable de interés para los inversores, lo que puede entenderse vinculado al hecho de que esta cifra contable no expresa el valor del intangible, excepto en el momento de la combinación de empresas. Tampoco resultó significativo el capital relacional, lo que es compresible si se tiene en cuenta, como indica Sveiby (1997), que se trata de una dimensión esencialmente externa, que es afectada también por factores ajenos a las empresas. Estos resultados están también en sintonía con los estudios previos, que no han aportado evidencia contundente en torno a la relevancia valorativa de ninguno de estos dos intangibles, tal como se refleja en los trabajos de Yu y Zhang (2008), Wang (2008), Kimouche y Rouabhi (2016b), Infante y Ferrer (2017) y Soetanto y Liem (2019). En este punto, es importante señalar que entendemos que estos resultados no contradicen las contribuciones antes señaladas a las teorías sobre las que se apoya este estudio, ya que, tal y como señalan Larrán y García (2004), existe información que es fundamental para valorar 
adecuadamente la empresa pero su utilidad para los usuarios está condicionada, tanto por su dificultad de medición como por su capacidad para ser extrapolada en la estimación de la cantidad y riesgo de los futuros flujos de caja de la empresa.

En este sentido, dada la propia configuración contable de estas variables, esta falta de relevancia valorativa que proporcionan los resultados del estudio, vendría a confirmar también que aquellas variables que no permiten reducir la asimetría de información y la incertidumbre -postulados básicos del papel asignado a la información por estas teorías-, son justamente las irrelevantes bajo sus postulados. Se pone así de manifiesto, una vez más, "una estrecha interrelación entre la economía de la información y la economía de la incertidumbre: la falta de información sobre las circunstancias relevantes del futuro o sobre el procedimiento para efectuar una valoración correcta de los resultados de las decisiones económicas, no es sino el reflejo de los problemas originados por la incertidumbre a que se enfrentan los agentes involucrados en las relaciones de producción e intercambio" (Azofra y Prieto, 1996, p. 46).

Finalmente, y en lo atinente a las variables contables fundamentales, TGPN y RDO, solo esta última resultó significativa. La falta de relevancia del patrimonio neto tangible resulta consistente con la que se le otorga al resultado y a los intangibles, en la medida que, como destacan Giner y Pardo (2007), el primero es un subrogado del valor de liquidación de la empresa, mientras que los demás conceptos son subrogados de futuros flujos de efectivo. Además, y tal como señalan Yu y Zhang (2008), quienes al igual que Oliveira et al. (2010) y Wahyuni et al. (2018) obtienen resultados coincidentes con los de esta investigación, la falta de relevancia valorativa del patrimonio neto tangible sugiere que los inversores priorizan el capital intangible por sobre el capital financiero como generador de valor.

\section{Conclusiones}

En este trabajo se analiza la relevancia de los intangibles para la formación de precios en el contexto del MCA, donde no se ha constatado la existencia de estudios previos de esta naturaleza. Hemos adoptado una perspectiva innovadora para el análisis que implica considerar tanto los intangibles reconocidos como los no reconocidos por la contabilidad, en relación a lo cual existe una importante laguna sin cubrir, en la medida que la investigación previa ha estudiado separadamente su relevancia valorativa, producto del abordaje desde dos campos diferentes: el de la investigación contable y el de la investigación en CI.

El estudio se realiza para el período 2009-2018 y sobre muestra de empresas pertenecientes a distintos sectores de actividad. Para poder concretarlo, y atender así al propósito de examinar -de manera conjunta- la relevancia valorativa de los AI y del CI, se ha construido un modelo de precios que incluye variables referidas a la diversidad intangibles involucrados en los dos grandes grupos antes referidos, lo que resulta novedoso desde el punto de vista metodológico. 
El modelo se basa en un marco de valoración de la empresa con sólidos fundamentos teóricos: el de Ohlson (1995), lo que también aporta al rigor de esta investigación, cuestión no siempre atendida en los trabajos previos, que muchas veces no han adoptado un modelo teórico que justifique la selección de las variables que se someten a análisis.

Los resultados obtenidos arrojan evidencia sobre la asociación entre el valor de las empresas cotizantes en el MCA y la información referida a los activos intangibles identificables y al capital humano y estructural, lo que permite confirmar la relevancia valorativa de estos intangibles.

Estos resultados muestran que los intangibles aportan información para la evaluación de las distintas oportunidades de inversión en el MCA y que contribuyen a la valoración de las empresas que participan del mismo. En particular, nuestros hallazgos denotan la capacidad de los inversores para valorar los distintos recursos intangibles basados en conocimiento como determinantes de la creación de valor de las empresas, al revelar que comprenden la importancia de los componentes estratégicos del CI: el capital humano, como componente primario y factor clave para su desarrollo, y el capital estructural, como sostén y elemento potenciador del factor humano e indicador fundamental de la aptitud de la organización para trasmitir y almacenar material intelectual.

La relevancia asignada a los activos intangibles identificables resulta consistente con lo anterior, en tanto involucran diversos elementos que revelan la capacidad de innovación de las empresas y que son el resultado acumulado de inversiones pasadas en generadores de valor que se han podido reconocer en los estados financieros. Además, la relevancia valorativa de los activos intangibles identificables sugiere que, a pesar de los problemas que plantea su contabilización, la cifra informada a través de dichos estados refleja el valor de sus beneficios económicos futuros, por lo que los inversores los perciben como activos legítimos capaces de proporcionar información relevante para la estimación del valor de la firma.

Estos hallazgos, que resultan coincidentes con las evidencias más sólidas que aportan los trabajos previos y a la vez apoyan los planteos de las teorías que sustentan el estudio, contribuyen a acrecentar la evidencia empírica existente en torno al papel que tienen los distintos tipos de recursos intangibles en la valoración de las empresas en los mercados de valores, la cual es necesaria para dar sustento a la construcción de una teoría, aún no desarrollada, que permita explicar la influencia de los intangibles en la creación de valor.

Además, dado que los estudios previos se han realizado sobre mercados de capitales con un importante grado de desarrollo, esta investigación, contextualizada en un mercado poco evolucionado como el argentino, contribuye a aumentar la limitada evidencia existente para este tipo de mercados.

Es preciso señalar también, como aportación del trabajo, su potencial para contribuir a la regulación contable, en la medida que los resultados obtenidos, que dan cuenta de la relevancia 


\section{C.R. Ficco, et al. / Contaduría y Administración 66 (3), 1-26 \\ http://dx.doi.org/10.22201/fca.24488410e.2021.2558}

de los intangibles para los inversores, contribuyen a hacer más patente la necesidad de incluir una mayor cantidad de información referida a estos recursos en los estados financieros, o en estados complementarios, en pos de incrementar su utilidad, lo que sirve al fortalecimiento de la base de conocimiento que podría orientar la elaboración de nuevas normas de contabilidad financiera o la modificación de las existentes.

No obstante, la investigación tiene ciertas limitaciones que es necesario dejar plasmadas. Por un lado, la escasa cantidad de empresas que integran la muestra, derivada de características inherentes al MCA. Este hecho, si bien ha permitido contar con información suficiente para estimar correctamente el modelo propuesto, condiciona algunos análisis adicionales que podrían aportar a una mejor comprensión del fenómeno estudiado. Entre ellos, el análisis por sectores, en tanto la aplicación de una clasificación sectorial representativa de la diversidad existente por tipo de actividad hubiera dado lugar a que quedaran sectores con muy pocas empresas. Por ello, de cara a una futura investigación, resultaría valioso realizar réplicas de este estudio sobre una muestra más amplia que incluya empresas de otros países, principalmente del entorno latinoamericano, lo que permitiría, además, indagar acerca de los efectos de las características culturales e institucionales en la relevancia valorativa de los intangibles.

Por otro lado, la falta de información no contable, públicamente disponible en Argentina, ha generado limitaciones a la hora de definir las variables a utilizar para medir el CI, lo que ha dado lugar a su medición empleando proxies basadas en medidas contables. En este sentido, teniendo en cuenta que ciertos indicadores no financieros podrían ser más representativos del CI, en una futura investigación se podría también explorar la posibilidad de construirlos a partir del análisis de la información sobre CI que las empresas revelan por medios diferentes a los estados financieros, entre los que cabría considerar a los informes de sostenibilidad.

Finalmente, otra interesante perspectiva futura es la relativa al análisis del efecto que puede tener la adopción de las NIIF en la relevancia valorativa de los intangibles, lo que aportaría a una comprensión más acabada de la relación entre los intangibles y la valoración de las empresas en el mercado.

\section{Referencias}

Andriessen, D. (2004). Making sense of intellectual capital: Designing a method for the valuation of intangibles. Burlington, USA: Elsevier Butterworth-Heinemann.

Asociación Española de Contabilidad y Administración de Empresas (2006). Documento No 8: Aplicabilidad del modelo de Ohlson para la valoración de acciones. Madrid: AECA.

Azofra, V., Ochoa, M., Prieto, B. y Santidrián, A. (2017). Creando valor mediante la aplicación de modelos de capital intelectual. Innovar, 27(65), 25-38. https://doi.org/10.15446/innovar.v27n65.64887

Azofra, V. y Prieto, M. B. (1996). La teoría positiva de la contabilidad en los sistemas de información contable internos. Madrid: ICAC - MEyH.

Badu, B. y Appiah, K. (2018). Value relevance of accounting information: an emerging country perspective. Journal of Accounting \& Organizational Change, 14(4), 473-491. https://doi.org/10.1108/jaoc-07-2017-0064 


\section{C.R. Ficco, et al. / Contaduría y Administración 66 (3), 1-26 \\ http://dx.doi.org/10.22201/fca.24488410e.2021.2558}

Baltagi, B. (2013). Econometric Analysis of Panel Data. (5th Ed.). New York: John Wiley \& Sons.

Barbosa, J., Coelho, A. y Weersma, L. (2019). The mediating effect of strategic orientation, innovation capabilities and managerial capabilities among exploration and exploitation, competitive advantage and firm's performance. Contaduría y Administración, 64(1), 1-25. http://doi.org/10.22201/fca.24488410e.2019.1918

Barney, J. (1991). Firm resources and sustained competitive advantage. Journal of Management, 17(1), 99-120. https://doi.org/10.1177/014920639101700108

Barth, M., Beaver, W. \& Landsman, W. (2001). The relevance of the value relevance literature for financial accounting standard setting: another view. Journal of Accounting and Economics, 31(1-3), 77-104. https://doi. $\operatorname{org} / 10.2139 / \mathrm{ssrn} .246861$

Barth, M. y Clinch, G. (2009). Scale effects in capital markets-based accounting research. Journal of Business Finance \& Accounting, 36(3-4), 253-288. http://doi.org/10.1111/j.1468-5957.2009.02133.x

Bayraktaroglu, A., Calisir, F. y Baskak, M. (2019). Intellectual capital and firm performance: An extended VAIC model. Journal of Intellectual Capital, 20(3), 406-425. https://doi.org/10.1108/jic-12-2017-0184

Bepari, M. y Mollik, A. (2017). Regime change in the accounting for goodwill: Goodwill write-offs and the value relevance of older goodwill. International Journal of Accounting \& Information Management, 25(1), 43-69. https://doi.org/10.1108/ijaim-02-2016-0018

Bollen, L., Vergauwen, P. y Schnieders, S. (2005). Linking intellectual capital and intellectual property to company performance. Management Decision, 43(9), 1161-1185. https://doi.org/10.1108/00251740510626254

Bontis, N. (1998). Intellectual capital: An exploratory study that develops measures and models. Management Decision, 36(2), 63-76. https://doi.org/10.1108/00251749810204142

Bontis, N., Chong, W. y Richardson, S. (2000). Intellectual capital and business performance in Malaysian industries. Journal of Intellectual Capital, 1(1), 85-100. https://doi.org/10.1108/14691930010324188

Cabrita, M. y Bontis, N. (2008). Intellectual capital and business performance in the Portuguese banking industry. International Journal of Technology Management, 43(1-3), 212-237. https://doi.org/10.1504/ijtm.2008.019416

Cameron, A. y Trivedi, P. (2005). Microeconometrics: Methods and Applications. New York: Cambridge University Press.

Cañibano, L., Sánchez, M. García, M. y Chaminade, C. (2002). Proyecto Meritum: Directrices para la gestión y difusión de información sobre intangibles. Madrid: Fundación Airtel Móvil.

Choi, W., Kwon, S. y Lobo, G. (2000). Market valuation of intangible assets. Journal of Business Research, 49(1), 35-45. https://doi.org/10.1016/s0148-2963(98)00121-0

Da Silva, A., Rodrigues, T. y Klann, R. (2017). A influência dos ativos intangíveis na relevância da informação contábil. Revista Contemporânea de Contabilidade, 14(31), 26-45. https://doi.org/10.5007/2175-8069.2017v $14 \mathrm{n} 31 \mathrm{p} 26$

Dahmash, F., Durand, R. y Watson, J. (2009). The value relevance and reliability of reported goodwill and identifiable intangible assets. The British Accounting Review, 41(2), 120-137. https://doi.org/10.1016/j.bar.2009.03.002

Dapena, J. (2012). Instrumentos de Inversión y Mercados Financieros. Buenos Aires: Fundación BCBA.

De Jager, P. (2008). Panel data techniques and accounting research. Meditari Accountancy Research, 16(2), 53-68. https://doi.org/10.1108/10222529200800012

Díaz, M. y Vargas, J. (2017). Estrategias metodológicas para datos de panel. El caso de los bancos típicos en Argentina. Ponencia, Congreso Interamericano de Estadística, Universidad Nacional de Rosario, Argentina.

Edvinsson, L. y Malone, M. (1997). Intellectual Capital. Realizing your company's true value by findings its hidden brainpower. New York: Harper Collins Publishers.

Ellis, H. y Seng, D. (2015). The value relevance of voluntary intellectual capital disclosure: New Zealand evidence. Corporate Ownership and Control, 13(1), 1071-1087. https://doi.org/10.22495/cocv13i1c9p9

Ely, K. y Waymire, G. (1999). Accounting standard-setting organizations and earnings relevance: Longitudinal evidence from NYSE common stocks, 1927-93. Journal of Accounting Research, 37(2), 293-317. https://doi. org/10.2307/2491411

Federación Argentina de Consejos Profesionales de Ciencias Económicas (2020). Resoluciones Técnicas vigentes. Buenos Aires: FACPCE. 


\section{C.R. Ficco, et al. / Contaduría y Administración 66 (3), 1-26 \\ http://dx.doi.org/10.22201/fca.24488410e.2021.2558}

Ferraro, O. y Veltri, S. (2011). The value relevance of intellectual capital on the firm's market value: An empirical survey on the Italian listed firms. International Journal of Knowledge-Based Development, 2(1), 66-84. https:// doi.org/10.1504/ijkbd.2011.040626

Ficco, C. (2019). Los activos intangibles en la normativa contable argentina y en las normas internacionales de información financiera. Contabilidad y Auditoría, 50, 61-108. Disponible en: http://www.ojs.econ.uba.ar/index. php/Contyaudit/article/view/1591/2247C. Consultado: 19/10/2019.

Forte, W., Matonti, G. y Nicolò, G. (2019). The impact of intellectual capital on firms' financial performance and market value: Empirical evidence from Italian listed firms. African Journal of Business Management, 13(5), 147-159. https://doi.org/10.5897/ajbm2018.8725

Forte, W., Tucker, J., Matonti, G. y Nicolò, G. (2017). Measuring the intellectual capital of Italian listed companies. Journal of Intellectual Capital, 18(4), 710-732. https://doi.org/10.1108/jic-08-2016-0083

Freeman, R. E. (1984). Strategic management: A stakeholder approach. Boston: Pitman.

García, L., Rodríguez, A. y García, J. (2018). Impact of investments in training and advertising on the market value relevance of a company's intangibles: The effect of the economic crisis in Spain. European Research on Management and Business Economics, 24(1), 27-32. https://doi.org/10.1016/j.iedeen.2017.06.001

Giner, B. y Pardo, F. (2007). La relevancia del fondo de comercio y su amortización en el mercado de capitales: Una perspectiva europea. Revista Española de Financiación y Contabilidad, 134(36), 389-415. https://doi.org $/ 10.1080 / 02102412.2007 .10779625$

Ginesti, G., Caldarelli, A. y Zampella, A. (2018). Exploring the impact of intellectual capital on company reputation and performance. Journal of Intellectual Capital, 19(5), 915-934. https://doi.org/10.1108/jic-01-2018-0012

Goebel, V. (2015). Estimating a measure of intellectual capital value to test its determinants. Journal of Intellectual Capital, 16(1), 101-120. https://doi.org/10.1108/jic-12-2013-0118

Gujarati, D. y Porter, D. (2010). Econometría. (Quinta Ed.). México: Mc Graw Hill.

Hail, L. (2013). Financial reporting and firm valuation: Relevance lost or relevance regained? Accounting and Business Research, 43(4), 329-358. https://doi.org/10.1080/00014788.2013.799402

Iazzolino, G., Chiappetta F. y Chiappetta, S. (2018). Relational Capital and financial performance: an empirical analysis on a simple of Italian firms. Problems and Perspectives in Management, 16(1), 245-258. https://doi. org/10.21511/ppm.16(1).2018.24

Infante, R y Ferrer, R. (2017). The impact of intangibles on the value relevance of accounting information: An empirical examination of listed companies in the Philippines from 2012 to 2016, Proceedings of the International Conference (October 2017), IGBR, ed.: 30-35.

International Accounting Standards Board (2018). Marco Conceptual para la Información Financiera. London: IASB.

International Accounting Standards Board (2018). Norma Internacional de Contabilidad $\mathrm{N}^{\circ}$ 38: Activos intangibles. London: IASB.

International Accounting Standards Board (2018). Norma Internacional de Información Financiera $N^{\circ} 3$ : Combinaciones de negocios. London: IASB.

Iñiguez, R. y López, G. (2005). Valoración de los activos intangibles en el mercado de capitales español. Revista Española de Financiación y Contabilidad, 34(125), 459-499. https://doi.org/10.1080/02102412.2005.10779553

Jensen, M. y Meckling, W. (1976). Theory of the firm: Managerial behaviour, agency costs and capital structure. Journal of Financial Economics, 3(4), 305-360. https://doi.org/10.2139/ssrn.94043

Kimouche, B. y Rouabhi, A. (2016a). The impact of intangibles on the value relevance of accounting information: Evidence from French companies. Intangible Capital, 12(2), 506-529. http://doi.org/10.3926/ic.653

Kimouche, B. y Rouabhi, A. (2016b). Intangibles and value relevance of accounting information: Evidence from UK companies. Jordan Journal of Business Administration, 12(2), 437-458. https://doi.org/10.12816/0033358

Kwon, S. y Wang, G. (2020). The change in the value relevance of accounting information after mergers and acquisitions: evidence from the adoption of SFAS 141 (R). Accounting \& Finance, 60(3), 2717-2757. https://doi. org/10.1111/acfi.12411 
Larrán, M. y García, E. (2004). La relevancia de la información no financiera en la estrategia empresarial de divulgación voluntaria: Percepciones empresa-analista sobre su utilidad. Revista valenciana de economía y hacienda, 12, 127-145. Disponible en: https://www.researchgate.net/publication/28105136_La_relevancia_ de_la_informacion_no-financiera_en_la_estrategia_empresarial_de_divulgacion_unitaria_Percepciones_empresa-analista_sobre_su_utilidad. Consultado: 15/04/2019.

Lev, B. (2001). Intangibles: management, measurement and reporting. Washington: The Brookings Institution Press.

Lev, B., Radhakrishnan, S. y Evans, P. (2016). Organizational capital. A CEO's guide to measuring and managing enterprise intangibles, Report, The Center for Global Enterprise.

Lev, B., Radhakrishnan, S. y Zhang, W. (2009). Organization capital. Abacus, 45(3), 275-298. https://doi.or$\mathrm{g} / 10.1111 / \mathrm{j} .1467-6281.2009 .00289 . x$

Lev, B. y Zarowin, P. (1999). The boundaries of financial reporting and how to extend them. Journal of Accounting Research, 37(2), 353-385. https://doi.org/10.2307/2491413

Liu, D., Tseng, K. y Yen, S. (2009). The incremental impact of intellectual capital on value creation. Journal of Intellectual Capital, 10(2), 260-276. https://doi.org/10.1108/14691930910952650

Mačerinskienė, I. y Survilaitè, S. (2019). Company's intellectual capital impact on market value of Baltic countries listed enterprises. Oeconomia Copernicana, 10(2), 309-339. https://doi.org/10.24136/oc.2019.016

Nimtrakoon, S. (2015). The relationship between intellectual capital, firms' market value and financial performance: Empirical evidence from the ASEAN. Journal of Intellectual Capital, 16(3), 587-618. https://doi. org/10.1108/jic-09-2014-0104

Nonaka, I. y Takeuchi, H. (1995). The knowledge creating company: How Japanese companies manage the dynamics of innovation. New York: Oxford University Press.

Ocak, M. y Findik, D. (2019). The impact of intangible assets and sub-components of intangible assets on sustainable growth and firm value: Evidence from Turkish listed firms. Sustainability, 11(19), 5359. https://doi. org/10.3390/su11195359

Ochoa, M., Prieto, M. B. y Santidrián, A. (2012). Una revisión de las principales teorías aplicables al CI. Revista Nacional de Administración, 3(2), 35-48. https://doi.org/10.22458/rna.v3i2.486

Ochoa, M., Prieto, M. B. y Santidrián, A. (2010). Estado actual de los modelos de capital intelectual y su impacto en la creación de valor en empresas de Castilla y León. Revista de Investigación Económica y Social de Castilla y León, 13, 15-205. Disponible en: https://www.cescyl.es/es/publicaciones/premios/actual-modelos-capital-intelectual-impacto-creacion-valor-e. Consultado: 08/01/2019.

Ohlson, J. (1995). Earnings, book values and dividends in equity valuation. Contemporany Accounting Research, 11(2), 661-687. https://doi.org/10.1111/j.1911-3846.1995.tb00461.x

Oliveira, L., Rodrigues, L. y Craig, R. (2010). Intangible assets and value relevance: Evidence from the Portuguese stock exchange. The British Accounting Review, 42(4), 241-252. https://doi.org/10.1016/j.bar.2010.08.001

Omarjee, F., Yasseen, Y. y Mohamed, W. (2019). The value relevance of aged goodwill: A South African study. Southern African Business Review, 19, 1-23. https://doi.org/10.25159/1998-8125/3868

Özer, G. y Çam, İ. (2017). The moderating effect of human capital on innovation capital and firm market value relationship. An application on BIST. International Journal of Management Economics and Business, 2017 Special Issue, 512-522. https://doi.org/10.17130/ijmeb.2017icmeb1735469

Priotto, H., Quadro, M., Veteri, L. y Werbin, E. (2011). Los activos intangibles: Una prueba empírica en empresas argentinas. Ponencia, XVI Congreso Internacional de Contaduría, Administración e Informática, México.

Pulic, A. (2000). VAIC ${ }^{\mathrm{TM}}$ - an accounting tool for IC management. International Journal of Technology Management, 20(5-8), 702-714. https://doi.org/10.1504/ijtm.2000.002891

Rieg, R. y Vanini, U. (2015). Value-Relevance of Intangibles and intellectual capital Disclosure on market-tobook value ratio: A longitudinal multilevel regression of german DAX Firms. Working Paper. https://doi. org/10.2139/ssrn.2906636

Ríos, A., Torres, M., Tirado, P. y Carbonell, A. (2009). Stakeholders, intangibles y generación de valor en las empresas del IBEX-35: Una estimación mediante modelos de panel. Revista Española de Financiación y Contabilidad, 38(142), 239-263. https://doi.org/10.1080/02102412.2009.10779668 


\section{C.R. Ficco, et al. / Contaduría y Administración 66 (3), 1-26 \\ http://dx.doi.org/10.22201/fca.24488410e.2021.2558}

Ritter, A. y Wells, P. (2006). Identifiable intangible asset disclosures, stock prices and future earnings. Accounting \& Finance, 46(5), 843-863. https://doi.org/10.1111/j.1467-629x.2006.00190.x

Roos, G. (2017). Knowledge management, intellectual capital, structural holes, economic complexity and national prosperity. Journal of Intellectual Capital, 18(4), 745-770. https://doi.org/10.1108/jic-07-2016-0072

Roos, G., Roos, J., Dragonetti, N. y Edvinsson, L. (2001). Capital Intelectual. Buenos Aires: Paidós.

Ross, S. A. (1979). Disclosure regulation in the financial markets: Implications of modern finance theory and signalling theory. New York: Mc Graw Hill.

Sardo, F. y Serrasqueiro, Z. (2018). Intellectual capital, growth opportunities, and financial performance in European firms: Dynamic panel data analysis. Journal of Intellectual Capital, 19(4), 747-767. https://doi. org/10.1108/jic-07-2017-0099

Sardo, F. y Serrasqueiro, Z. (2017). A European empirical study of the relationship between firms' intellectual capital, financial performance and market value. Journal of Intellectual Capital, 18(4), 771-788. https://doi. org/10.1108/jic-10-2016-0105

Scott, W. (2009). Financial Accounting Theory. (5th Ed.). Toronto: Pearson Prentice Hall.

Sharma, P. (2018). Enterprise value and intellectual capital: Study of BSE 500 firms. Accounting and Finance Research, 7(2), 123-133. https://doi.org/10.5430/afr.v7n2p123

Smriti, N. y Das, N. (2018). The impact of intellectual capital on firm performance: A study of Indian firms listed in COSPI. Journal of Intellectual Capital, 19(5), 935-964. https://doi.org/10.1108/JIC-11-2017-0156

Soetanto, T. y Liem, P. (2019). Intellectual capital in Indonesia: Dynamic panel approach. Journal of Asia Business Studies, 13(2), 240-262. https://doi.org/10.1108/jabs-02-2018-0059

Suherman, R. (2017). The impact of intellectual capital toward firm's profitability and market value of retail companies listed in Indonesia Stock Exchange (IDX) from 2013-2016. iBuss Management, 5(1), 98-112. Disponible en: http://publication.petra.ac.id/index.php/ibm/article/view/5877. Consultado: 08/01/2019.

Sveiby, K. (1997). The intangible assets monitor. Disponible en: https://www.sveiby.com/article/The-Intangible-Assets-Monitor. Consultado: 08/01/2019.

Sveiby, K. (2018). Measuring intangibles: suggested indicators with cases. Disponible en: https://www.sveiby.com/ article/Measuring-Intangibles-Suggested-Indicators-with-Cases. Consultado: 08/01/2019.

Swartz, G., Swartz, N. y Firer, S. (2006). An empirical examination of the value relevance of intellectual capital using the Ohlson (1995) valuation model. Meditari Accountancy Research, 14(2), 67-81. https://doi. org/10.1108/10222529200600013

Tejedo, F. y Alfaro, E. (2014). Asociación entre las características empresariales y la divulgación sobre capital intelectual: Un estudio de las empresas del Ibex 35. Estudios de Economía Aplicada, 32(1), 371-398. https:// doi.org/10.25115/eea.v32i1.3215

Tejedo, F. y Araujo, J. (2018). Estrategia de gestión y divulgación del capital intelectual: Influencia del gobierno corporativo. Contaduría y Administración, 63(2), 1-18. https://doi.org/10.22201/fca.24488410e.2018.1236

Tejedo, F., Ferraz, J. y Emmendoerfer, M. (2017). Mecanismos de gobierno corporativo y capital intelectual. Revista Brasileira de Gestão e Negócios, 19(65), 394-414. https://doi.org/10.7819/rbgn.v19i65.3024

Tolosa, L. (2013). El contenido informativo de los estados contables y de los precios de las acciones en la toma de decisiones de inversión. Análisis de empresas que cotizan en el mercado de capitales argentino. Tesis doctoral, Universidad Nacional de Córdoba, Argentina.

Tyskbo, D. (2019). Managers' views on how intellectual capital is recognized and managed in practice: A multiple case study of four Swedish firms. Journal of Intellectual Capital, 20(2), 282-304. https://doi.org/10.1108/JIC01-2018-0017

Veltri, S. y Silvestri, A. (2011). Direct and indirect effects of human capital on firm value: Evidence from Italian companies. Journal of Human Resource Costing \& Accounting, 15(3), 232-254. https://doi. org/10.1108/14013381111178596

Villegas, E., Hernández, M. y Salazar, B. (2017). La medición del capital intelectual y su impacto en el rendimiento financiero en empresas del sector industrial en México. Contaduría y Administración, 62(1), 184-206. http:// doi.org/10.1016/j.cya.2016.10.002 


\section{C.R. Ficco, et al. / Contaduría y Administración 66 (3), 1-26}

http://dx.doi.org/10.22201/fca.24488410e.2021.2558

Wahyuni, E., Dewantoro y Avianti, I. (2018). Has goodwill become more relevant after IFRS convergence in Indonesia? Journal of Accounting and Investment, 19(2), 239-256. https://doi.org/10.18196/jai.1902104

Wang, J. (2008). Investigating market value and intellectual capital for SyP 500. Journal of Intellectual Capital, 9(4), 546-563. https://doi.org/10.1108/14691930810913159

Wernerfelt, B. (1984). A resource-based view of the firm. Strategic Management Journal, 5(2), 171-180. https:// doi.org/10.1002/smj.4250050207

White, H. (1980). A heteroskedasticity-consistent covariance matrix estimator and a direct test for heteroskedasticity. Econometrica, 48(4), 817-838. https://doi.org/10.2307/1912934

Whiting, R. y Miller, J. (2008). Voluntary disclosure of intellectual capital in New Zealand annual reports and the "hidden value". Journal of Human Resource Costing \& Accounting, 12(1), 26-50. https://doi. org/10.1108/14013380810872725

Xu, J. y Li, J. (2019). The impact of intellectual capital on SMEs' performance in China. Journal of Intellectual Capital, 20(4), 488-509. https://doi.org/10.1108/JIC-04-2018-0074

Xu, J. y Wang, B. (2018). Intellectual capital, financial performance and companies' sustainable growth: evidence from the Korean manufacturing industry. Sustainability, 10(12), 4651. https://doi.org/10.3390/su10124651

Yu, F. y Zhang, L. (2008). Does intellectual capital really create value? 4th International Conference on Wireless Communications, Networking and Mobile Computing. IEEE. https://doi.org/10.1109/wicom.2008.2324

Yu, H., Wang, W. y Chang, C. (2009). The pricing of intellectual capital in the IT industry, Working Paper, National Chengchi University. http://doi.org/10.2139/ssrn.1327668 